\title{
Körperlich-seelische Berührungen im Fokus der Psychoneuroimmunologie
}

\author{
Michaela Ott, Magdalena Singer, Karl Heinz Brisch, Christian Schubert
}

\begin{abstract}
Die Psychoneuroimmunologie zeigt, dass Berührungs- und Bindungserfahrungen pränatal und in der frühen Kindheit unser Stresserleben und -verhalten bis ins hohe Alter „programmieren“. Infolgedessen leiden körperlich und seelisch traumatisierte Kinder später öfters unter entzündungsassoziierten Krankheiten und chronischen Schmerzen. Die Forschung belegt weiter, dass Berührungstraumen und der damit verbundene Schmerz überwunden werden können.
\end{abstract}

\section{Hintergrund}

Als der Körperkontakt in amerikanischen „Findelhäusern“ Anfang des 20. Jahrhunderts aus hygienischen Gründen eingeschränkt war, machte man die schreckliche Beobachtung, dass Kinder unter einem Jahr häufiger verstarben [70][71]. Es gilt als wahrscheinlich, dass die Findelkinder nicht allein wegen eines Mangels an körperlichtaktiler Berührung durch die Pflegekräfte starben, sondern auch wegen der damit einhergehenden emotionalen Vernachlässigung [44][64][84]. Das historische Beispiel zeigt, dass Neugeborene maßgeblich auf das Berührt-Werden angewiesen sind. Gleichzeitig wird deutlich, dass der Begriff „Berührung“ neben seiner mechanisch-körperlichen Komponente auch die Dimensionen des emotionalen „Berührt-Seins“ und sozialen „Berührt-Werdens“ umfasst [22]. Dabei ist das Berühren reziproker Natur: Auch Kinder berühren ihre Eltern emotional (z. B. „Kindchenschema“, „soziales Lächeln“). So festigen sie die Bindung zu ihren Bezugspersonen und sichern sich deren Fürsorge [39].

Die Mehrdimensionalität von Berührung wird am ehesten durch das biopsychosoziale Modell (BPS-Modell) erfasst [32]. George Engels systemtheoretische Konzeption aus dem Jahre 1980 betrachtet das komplexe Wechselspiel biologischer, psychischer und sozialer Faktoren als ausschlaggebend für die Person und ihre Gesundheit oder Krankheit eines Menschen. Dabei haben höher komplexe soziale Faktoren (Umwelt, Nation, Kultur, Gesellschaft, Vertrauenspersonen, Freunde, Familie, Partnerschaft, etc.) mehr Einfluss auf die Gesundheit (Wirkung von oben nach unten bzw. top-down) als weniger komplexe biologische Faktoren (Nerven- und
Organsysteme, Gewebe, Zellen etc.) (Wirkung von unten nach oben bzw. bottom-up)[79].

\section{Psychoneuroimmunologie}

Die Psychoneuroimmunologie (PNI) gilt als mögliche empirische Realisierung des BPS-Modells [79]. Sie untersucht die Wechselwirkungen zwischen sozialen bzw. psychischen Faktoren und dem Nerven-, Hormon- und Immunsystem [77]. Aktuelle PNI-Forschungen berücksichtigen, dass Eltern die Umwelt ihrer Kinder bestimmen, deren psychosoziale Entwicklung beeinflussen und zu deren langfristiger Gesundheit maßgeblich beitragen. Die Untersuchungen fokussieren auf das gesamte Lebensspannenthema von der pränatalen Entwicklung bis ins hohe Alter.

\section{Grundlagen}

Immun-, Nerven- und Hormonsystem bilden gemeinsam das „immuno-neuro-endokrine Netzwerk“, ein Stressregulationssystem, das über Gesundheit oder Krankheit entscheidet [8]. Die Kommunikation zwischen den Systemen vollzieht sich über eine "gemeinsame biochemische Sprache“ [10].

Bei Angst, Wut, Hunger, Schmerz oder ähnlichen stressassoziierten seelischen Zuständen reagieren v.a. das Nerven-, das Hormon- sowie das Immunsystem. Bei einem gesunden Menschen imponiert bei akutem Stress ein kurzfristiger Anstieg der Entzündungsparameter [9]. Um ein Überschießen der stressbedingten Entzündungsreaktion zu verhindern, werden bei akuten Entzündungsantworten zeitgleich entzündungsregulierende 
Prozesse (z.B. Ausschüttung von Kortisol) in Gang gesetzt [8]. Bei chronischem Stress werden diese Regelmechanismen überbeansprucht (z. B. Hypercortisolismus, Hypocortisolismus [86]). Dadurch kommt es zu Immundysbalancen und überhöhten Entzündungsanstiegen [29][30] sowie dem Auftreten von stressassoziierten Krankheitssymptomen (z.B. Erschöpfung, Schmerzen, Depressivität) [25] ( Tab. 1).

Die Ursachen für diese entzündungsassoziierten neurovegetativen und neuropsychiatrischen Beschwerden können in der vorgeburtlichen und frühkindlichen Entwicklung liegen. Sie stehen, wie die folgenden Abschnitte zeigen werden, häufig in Zusammenhang mit mangelnden oder fehlgeleiteten körperlich-seelischen Berührungserfahrungen wie Gewalt, Missbrauch oder Vernachlässigung [24][53].

\section{Berührungserfahrungen}

\section{Pränatale Kommunikation}

Körperliche Berührungen werden v. a. über die Haut als größtem Sinnesorgan wahrgenommen. Der Tastsinn des Ungeborenen entwickelt sich vor allen anderen Sinnen in der 8. Schwangerschaftswoche (SSW) [41]. Spürt das Kind Berührungsreize im Mutterleib, bewegt es sich [60]. Diese Bewegungen werden von der Mutter etwa ab der 16.-20. SSW wahrgenommen [1].

Die „feto-maternale Schnittstelle“ zwischen Mutter und Kind dient als zentraler Kommunikationsweg [62]: Über die Nabelschnur empfängt der Fetus die Botschaften und Signale aus der mütterlichen Gefühlswelt und reagiert dabei sehr sensitiv auf Umwelt- und Stresseinflüsse [6] [69][87]. Bestenfalls sorgen psychoneuroimmunologische Schutzprozesse für die gesunde Entwicklung des Ungeborenen [83]. So verändert sich das Immunsystem der Schwangeren, um den Fetus vor gefährlichen Entzündungsanstiegen zu schützen: Es imponiert eine antiinflammatorische Immunlage (TH2-Dominanz) sowie ein protektiver Anstieg entzündungshemmender Hormone (Progesteron, Kortisol) [83]. Da aber zu viel Kortisol für die Entwicklung des Fetus schädlich ist, vollzieht sich gleichzeitig über die „feto-maternale Schnittstelle“ [62] ein maßvoller Kortisol-Transfer zum Kind [80][81].

FETAL PROGRAMIMING

Fetal Programming beschreibt, wie intensiver und andauernder vorgeburtlicher Stress beim ungeborenen Kind zur Fehlprogrammierung von Organsystemen und Stoffwechselprozessen führen kann. Fetal Programming betrifft jedoch auch positive Effekte und somit die pränatale Prägung der Gesundheit [72].

\section{Berührungserfahrungen der Schwangeren}

Um zu klären, welche Auswirkung die körperlich-seelischen Berührungserfahrungen während der Schwangerschaft auf das Ungeborene haben, muss der Blick unweigerlich auf die Mutter und ihre Lebensrealität (z. B. zwischenmenschliche Beziehungen) gelenkt werden [3] ( Tab. 1).

\section{Positive Aspekte}

Unzweifelhaft tragen positive Berührungserfahrungen der werdenden Mutter zum psychoneuroimmunologischen Gelingen der Schwangerschaft und zum Gedeihen des Fetus bei. Auch die soziale Unterstützung der Schwangeren gilt als positiver Aspekt körperlich-seelischer Berührung.

Positive Berührungserfahrungen wie die soziale Unterstützung dienen der Schwangeren und ihrem Ungeborenen als „Stresspuffer“ [57]. Sie sorgen für das optimale Wachstum des Feten korrelieren mit einem höheren Geburtsgewicht [27]. Je positiver Schwangere im dritten Trimester die Beziehungsqualität ihrer Partnerschaft einschätzten, desto höher waren die antientzündlichen IL10-Werte [67].

\section{Negative Aspekte}

Negative Berührungserfahrungen wie z. B. als mangelhaft erlebte soziale Unterstützung der Schwangeren sowie starkes Stresserleben stören die physiologischen Anpassungsprozesse des Ungeborenen und gefährdet dessen Entwicklung. Schwangere, die im zweiten und dritten Trimester über viel Stress und wenig soziale Unterstützung berichteten, wiesen höhere Werte des unspezifischen Entzündungsparameters CRP auf, als Frauen, die in dieser Zeit mehr soziale Unterstützung erfuhren. Höhere Entzündungswerte erhöhen das Risiko für Schwangerschaftskomplikationen (z. B. Präeklampsie, Frühgeburt) [23].

Der anhaltende Entzündungsanstieg sowie der übermäßige Kortisol-Transfer zum Kind haben gesundheitliche Folgen für den Fetus: Sie beeinträchtigen die Hirnentwicklung, führen zur Dysregulation der HPA-Achse und stören die Immunentwicklung [63].

\section{Spätfolgen}

Eine pränatale Dysregulation des Stresssystems kann sich über die Geburt hinaus sogar bis ins Erwachsenenalter fortsetzen und so das Risiko für diverse Entzündungsund Immunerkrankungen erhöhen [53].

Ein wegweisendes Langzeitprojekt untersuchte zwei Gruppen junger Erwachsener, deren Mütter während der Schwangerschaft entweder durch Trennung, Tod, Krankheit, finanzielle Sorgen etc. betroffen waren oder aber keine belastenden Lebensereignisse erlebten. Die Untersuchung zeigte, dass diese aversiven Berührungserfahrungen der Schwangeren die Entwicklung einer relativen 
- Tab. 1 Stressreaktionen von Vegetativum, Hormon- und Immunsystem, physiologische Gegenregulationen sowie Pathologien bei Dysregulation des „immuno-neuro-endokrinen Netzwerks“.

\begin{tabular}{|c|c|c|c|c|}
\hline Stressreaktion & Nervensystem & Hormonsystem & Immunsystem & Krankheitszeichen \\
\hline $\begin{array}{l}\text { Physiologische Stressreak- } \\
\text { tion bei Angst, Wut, Hun- } \\
\text { ger, Schmerz etc. }\end{array}$ & $\begin{array}{l}\text { Sympathikus getriggerte Steige- } \\
\text { rung der Aktionsfähigkeit bei tat- } \\
\text { sächlicher oder gefühlter } \\
\text { Belastung („Fight-or-Flight“) } \\
\text { [21][86] } \\
\text { - Erhöhen der Durchblutung der } \\
\text { Muskulatur } \\
\text { - Steigerung der Aktivität von } \\
\text { Herz- und Atmungsorganen } \\
\text { - Drosselung nicht überlebens- } \\
\text { wichtiger } \\
\text { Funktionen, z. B.: } \\
\text { - Verdauungstätigkeit } \\
\text { - Fortpflanzungsaktivität }\end{array}$ & & 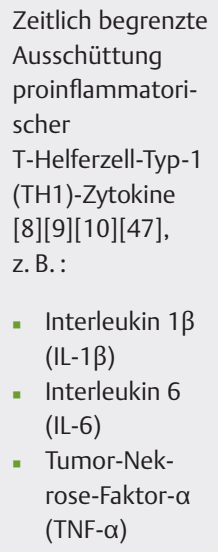 & \\
\hline $\begin{array}{l}\text { Physiologische Gegen- } \\
\text { regulation (vs. überschie- } \\
\text { ßende Entzündung) }\end{array}$ & $\begin{array}{l}\text { "anti-inflammatorischer Reflex“ } \\
\text { des Parasympathikus [85] }\end{array}$ & $\begin{array}{l}\text { Ausschüttung des } \\
\text { anti-inflammatori- } \\
\text { schen Hormons } \\
\text { Kortisol via Hypo- } \\
\text { thalamus-Hypo- } \\
\text { physen-Nebennie- } \\
\text { renrinden-Achse } \\
\text { (hypothalamic- } \\
\text { pituitary-adreno- } \\
\text { cortical, HPA- } \\
\text { Achse) [86] }\end{array}$ & $\begin{array}{l}\text { Verschieben der } \\
\text { Immunbalance } \\
\text { (TH1 / TH2-Shift) } \\
\text { von pro-inflam- } \\
\text { matorischen TH-1 } \\
\text { zu anti-inflamma- } \\
\text { torischen T-Hel- } \\
\text { ferzell-Typ-2 } \\
\text { (TH2)-Zytokine } \\
\text { [29][30], z. B. : } \\
\text { Interleukin } 4 \\
\text { (IL-4) } \\
\text { - Interleukin } 10 \\
\text { (IL-10) }\end{array}$ & \\
\hline \multirow[t]{2}{*}{$\begin{array}{l}\text { Pathologische Stressreak- } \\
\text { tion bei Dysregulation des } \\
\text { „immuno-neuro-endokri- } \\
\text { nen Netzwerks“ }\end{array}$} & & $\begin{array}{l}\text { - Hypercortiso- } \\
\text { lismus: } \\
\text { - nach lang- } \\
\text { dauernder } \\
\text { psychi- } \\
\text { scher } \\
\text { Belastung } \\
\text { [86] }\end{array}$ & $\begin{array}{l}\text { chronischer TH1 / } \\
\text { TH2-Shift mit } \\
\text { dauerhaft } \\
\text { - erniedrigten } \\
\text { TH1-Zytokinen } \\
\text { (verringerter } \\
\text { Immunschutz) } \\
\text { - erhöhten TH2- } \\
\text { Zytokinen } \\
\text { (proallergene } \\
\text { Immunlage) } \\
\text { [29][30] }\end{array}$ & $\begin{array}{l}\text { - gehäuftes Auftreten von z. B. } \\
\text { - Infektionskrankheiten, } \\
\text { Krebs (TH1 schützt) } \\
\text { - Allergien (exzessive TH2- } \\
\text { Reaktion) [29][30] }\end{array}$ \\
\hline & & $\begin{array}{l}\text { - Hypocortisolis- } \\
\text { mus: } \\
\text { - nach } \\
\text { Traumata } \\
\text { [86] }\end{array}$ & $\begin{array}{l}\text { chronisch erhöhte } \\
\text { Entzündungsle- } \\
\text { vels können zu } \\
\text { Zellschädigungen } \\
\text { und -entartungen } \\
\text { führen [29][30] }\end{array}$ & $\begin{array}{l}\text { " höheres Risiko für Entzün- } \\
\text { dungsreaktionen } \\
\text { (z. B. Autoimmunkrankheiten, } \\
\text { kardiovaskuläre und pulmo- } \\
\text { nale Erkrankungen) (exzessive } \\
\text { TH1-Reaktion) [29][30] } \\
\text { - Sickness Behavior [25]: } \\
\text { - Erschöpfung } \\
\text { - Konzentrationsstörungen } \\
\text { - Schmerzen }\end{array}$ \\
\hline
\end{tabular}


Insulinresistenz, Dysregulationen in der HPA-Achse sowie ein erhöhtes TH2-Zytokin-Profil bei den mittlerweile erwachsenen Kindern bedingten [33][34][35]. Erhöhte TH2-Zytokinkonzentrationen lassen das Risiko für atopische Erkrankungen (z. B. allergisches Asthma bronchiale) und Autoimmunerkrankungen ansteigen (Tab. 1) [29][75].

Jüngste Forschungen legen zudem nahe, dass negative Berührungserfahrungen der Mutter in ihrer eigenen Kindheit (z. B. Misshandlungen) an an die nächste Generation weitergegeben werden können. Die Schwangerschaft dürfte dabei eine besonders kritische Phase darstellen [20][56].

\section{Postnatale Berührungserfahrungen}

\section{Berührung als Grundbedürfnis}

Nach der Geburt spielt die Qualität körperlich-seelischer Berührungen eine entscheidende Rolle für die gesunde körperliche, psychische und soziale Entwicklung des Kindes [14][37]. Frühe Berührungserfahrungen wie das Stillen bzw. körpernahe Füttern, das Tragen, die achtsame Körperpflege, das geborgene Schlafen in unmittelbarer Nähe zu Bezugspersonen sowie die Babymassage sind von elementarer Wichtigkeit, da sie dem Kind ein Urvertrauen vermitteln [54].

\section{Berührungseffekte}

Die direkte körperliche Nähe eines Kindes zu seinen Bezugspersonen dürfte sich beruhigend auf dessen „immuno-neuro-endokrines Netzwerk“ in seiner Funktion als Stressregulationssystem auswirken. Eine aktuelle Übersichtsarbeit zeigt den positiven Einfluss der Massagetherapie auf das kindliche Stress- und Immunsystem ( Abb. 2) [38].

\section{Bindungserfahrungen}

Die überlebensnotwendige Bindung zwischen Bezugsperson und Säugling entsteht durch Liebe, Empathie, Versorgung und Kontakt. Die Organisation dieser Bindung erfolgt zu einem großen Teil über das Medium Berührung [3][12][26].

\section{Bindungsstile}

\section{Sicherer Bindungsstil}

Womöglich ist eine sichere Bindung zwischen Kind und Bezugspersonen der wesentliche Schutzfaktor für die gesunde Entwicklung des kindlichen Immunsystems [18][28][42][65]. Ein sicherer Bindungsstil ist gekennzeichnet von elterlicher Feinfühligkeit in der Interaktion mit dem Kind, prompter Wahrnehmung der kindlichen Signale sowie deren richtiger Interpretation. Sicher gebundene Kinder haben eine große Zuversicht in die Verfügbarkeit ihrer Bindungspersonen [18].
Positive Bindungserfahrungen scheinen sogar das Potenzial zu haben, negative gesundheitliche Konsequenzen widriger Lebensumstände abzupuffern, indem sie z. B. die Entstehung früher chronischer Entzündungsprozesse verhindern. Wie die Zusammenhänge zwischen Immunsystem und Bindungsorganisation vermittelt werden, ist bis dato aber nicht vollständig geklärt [65].

Sicher gebundene Kinder, die in ihrer Vergangenheit u. a. Armut, Misshandlung, Vernachlässigung, harschen Erziehungsmethoden oder psychischen Problemen der Eltern ausgesetzt waren, weisen im Gegensatz zu unsicher oder desorganisiert gebundenen Kindern mit ähnlich widrigen Kindheitserlebnissen niedrigere CRP-Entzündungslevels auf [7].

Bereits vor 30 Jahren wurde-anhand der Funktion der HPA-Achse-erforscht, wie sich der Bindungsstil bei Kindern auf deren Stressregulationssystem auswirkt [43]. Ergebnis: Wenn Kinder sicher an ihre Eltern gebunden sind, dürften diese bis zur Pubertät des Kindes als zentrale Stressregulatoren fungieren. Aufgrund dieses „Social Bufferings“ reagieren sicher gebundene Kinder etwa ab Beginn des 1. Lebensjahres bis zur Pubertät bei Stress (Impfung, Arztbesuch etc.) nicht mit Kortisol-Anstiegen [43].

\section{Unsicherer Bindungsstil}

Menschen mit unsicherem oder desorganisiertem Bindungsstil erlebten in ihrer Kindheit oftmals aversive oder mangelnde körperlich-seelische Berührungen [40][50]. Das dadurch in seiner Entwicklung gestörte Stresssystem imponiert im Erwachsenenalter oftmals mit Hypocortisolismus bzw. chronisch erhöhten Entzündungswerten [52][65][66][68]. Demgemäß zeigte eine Metaanalyse bei in der Kindheit bzw. Jugend traumatisierten

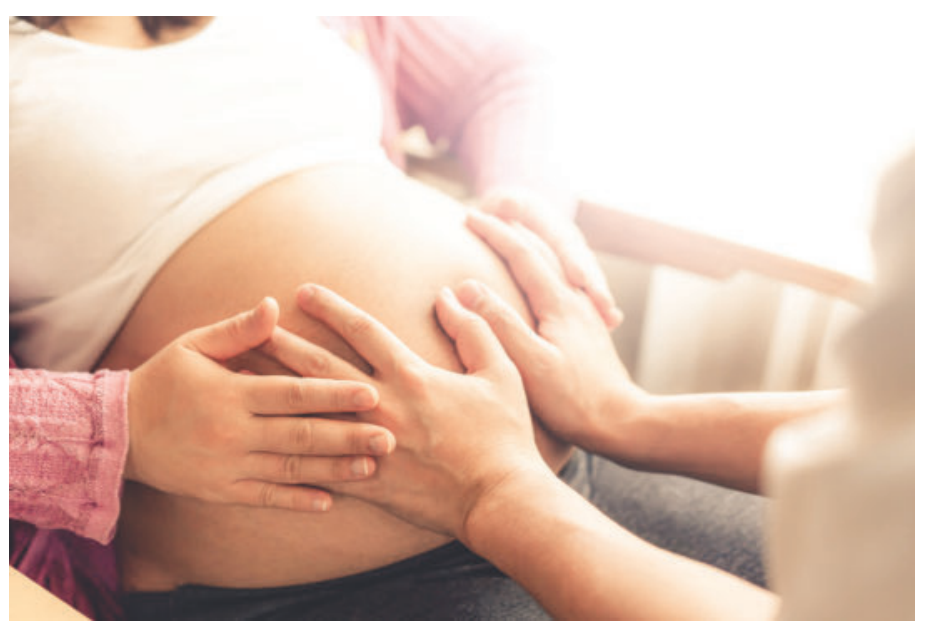

- Abb. 1 Das Berühren und Streicheln des Bauches als Reaktion auf die Bewegungen des Ungeborenen ist eine besondere Form der Kommunikation zwischen Kind und Außenwelt [54]. (Quelle: Blue Planet Studio stock.adobe.com (Symbolfoto). 
Erwachsenen signifikant höhere CRP-, IL-6- und v. a. TNF$\alpha$-Grundlevels [5]. Verantwortlich für diese Langzeitfolgen dürften Veränderungen in der epigenetischen Regulation der Genexpression in den Immunfunktionen sein. Ein pro-inflammatorisches Entzündungsprofil erhöht letztlich das Risiko für diverse psychische und körperliche Erkrankungen [5][36].

Merke

Berührung und Bindung

Entzündungsassoziierte Krankheiten wie Depressionen, Angst- und posttraumatische Belastungsstörungen, Psychosen oder auch rheumatoide Arthritis, kardiovaskuläre und pulmonale Erkrankungen, das metabolische Syndrom und schließlich auch Krebs können mit frühen Traumatisierungen in Verbindung stehen und verdeutlichen deshalb, wie dramatisch sich Einbußen im berührungsabhängigen Bindungsgeschehen über die gesamte Lebensspanne hinweg fortsetzen [5][36].

\section{Chronischer Schmerz}

Auch das Phänomen des chronischen Schmerzes wird mit widrigen Erfahrungen in der frühen Kindheit [45] [59] in Verbindung gebracht. Die Vita chronischer Schmerzpatienten ist häufig geprägt von schweren körperlichen und seelischen Schmerzen bzw. Gewalterfahrungen [4].

\section{Physis und Psyche}

Physische Gewalterfahrungen und psychische Schmerzen dürfen nicht dualistisch betrachtet werden. Ein Blick durch die „biopsychosoziale Brille“ [74] führt vor Augen, dass ein Mensch nicht nur ausschließlich körperlich oder seelisch verletzt wird. Die PNI untersucht, wie früh in der Biographie verankerte Traumata und Stresserfahrungen

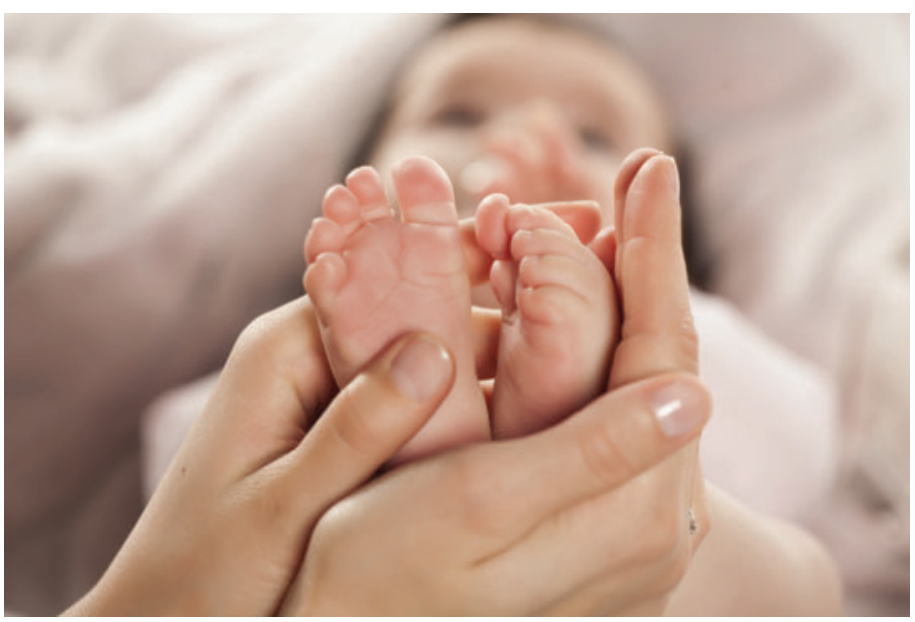

- Abb. 2 Das Touch Research Institute in Miami bestätigt der Massagetherapie gesundheitsförderliche Effekte bis ins hohe Lebensalter (Erhöhen der Wachsamkeit, Reduktion der Stresshormone, Verbesserung der Immunfunktion, Schmerzreduktion etc.) [38]. (Foto: vladimirfloyd stock.adobe.com, Symbolbild) später davon vermeintlich losgelöste Schmerzzustände provozieren können.

\section{Schmerzmodulation}

Moderne Schmerztheorien verstehen unter Schmerzmodulation Veränderungen der Schmerzwahrnehmung (Verringerung, Verstärkung), die immuno-neuro-endokrin vermittelt werden und in Abhängigkeit von aktuellen und vergangenen Umwelteinflüssen stehen [89].

Aus Tierversuchen ist bekannt, dass die bidirektionale Interaktion zwischen Immun- und Nervensystem ausschlaggebend für die Schmerzmodulation ist [89]. Wie erläutert, können widrige oder traumatische Lebensereignisse wie Gewalt oder Missbrauch bereits während Schwangerschaft, Geburt oder früher Kindheit die Stressregulation aus dem Gleichgewicht bringen. Der daraus letztendlich resultierende permanente Anstieg entzündungsfördernder Zytokine bedingt neurovegetative und neuropsychiatrische Beschwerden, darunter auch Schmerzen (,sickness behavior“) [25].

Langfristig erhöht die entzündungsassoziierte Sensibilisierung der Nozizeptoren auch die Schmerzsensitivität. Zudem aktiviert die bei Entzündungsstress stimulierte HPA-Achse neben medialem präfrontalem Kortex und Hippocampus auch die Amygdala und führt zur Schmerzsensibilisierung. Die angeführten stressgetriggerten und entzündungsassoziierten Mechanismen dürften - über daueraktivierte Mikroglia und andere „geprimte“ Zellen - die Grundlage für chronische Schmerzen darstellen [89].

\begin{abstract}
Merke
Therapie chronischer Schmerzpatienten

Bei der Behandlung chronischer Schmerzen müssen im Kontext aktueller als auch frühkindlicher Stresserfahrungen - psychoneuroimmunologische Wechselwirkungen miteinbezogen werden [89]. Die systematische Reduktion von Entzündung gilt als wesentliches Kriterium der Schmerzverbesserung und sollte sowohl topdown als auch bottom-up realisiert werden [58]. So reduzieren kontemplative Techniken, z. B. Meditation, unmittelbar das psychische Stresserleben und damit auch ,top-down' die körperliche Entzündungsaktivität, während andere, vorrangig körperliche Verfahren wie Physio- und Atemtherapie, Yoga, QiGong oder TaiChi über die kontrollierte Atemarbeit und über körperliche Praktiken ,bottom-up، Stresssystemfunktionen beeinflussen.
\end{abstract}

\section{Berührung in der Behandlung von Patienten}

Bei einem biopsychosozialen Verständnis der Begriffe „Berührung“ und „Schmerz“ spielen Berührungserfahrungen in nahezu jeder Heilmethode eine tragende Rolle-angefangen von der ärztlichen Visite über die Physio-, Massage- oder Psychotherapie bis hin zu 
Osteopathie und Mind-Body-Verfahren (Meditation, Klangschalentherapie, QiGong, etc.).

Die einzelnen Methoden setzen Berührungsreize unterschiedlich bewusst ein bzw. messen den Berührungselementen ein unterschiedliches Maß an Bedeutung bei. Wenn etwa ein unüberlegt agierender Arzt seinem Patienten eine negative Prognose wenig empathisch verkündet und ihn dabei negativ „berührt“, kann dieser Kontakt zum schädigenden (Nocebo-)Reiz werden [73].

Positive Berührungen können im Rahmen der BehandlerPatienten-Interaktion eine sichere Beziehung hervorrufen (hilfreiches „therapeutic-bonding“) [11][61] und/ oder in der Behandlung zielgerichtet eingesetzt werden. Zu den Methoden, in denen „berührt“ wird, um zu heilen, zählt die Osteopathie, auf die in Folge exemplarisch eingegangen wird [82].

\section{Beispiel: Osteopathie}

Das ganzheitliche Konzept der Osteopathie respektiert die Körper-Seele-Geist-Einheit jedes Menschen. Hauptmedium der Behandlung ist die Berührung [82]. Aus osteopathischer Sicht führen muskuloskelettale oder viszerale Bewegungseinschränkungen zunächst zu Gewebespannungen und dann zu Funktionsstörungen. Behandlungsziel ist das Wiederherstellen oder Bewahren der harmonischen Beweglichkeit des Körpers mit dem Ziel der Aktivierung von Selbstheilungskräften [88].

In einer Studie mit randomized controlled trial (RCT)Design wurden 70 Patienten mit unspezifischen Schmerzen im unteren Rücken wöchentlich osteopathisch behandelt. Bei den Testpersonen verbesserten sich die Rückenschmerzen signifikant; gleichzeitig sank die Konzentration des Entzündungsstoffes TNF- $\alpha$ [49]. Schmerzund Entzündungsreduktion dürften folglich in einem unmittelbaren Zusammenhang zueinander stehen.

\section{Beziehungsmedizinische Aspekte}

Berührungsassoziierte Verfahren wie die Osteopathie, Physio- und Massagetherapie scheinen auf den ersten Blick hin als „mechanistisch funktionierend“. Bei genauerer Betrachtung dürfte ihre Effizienz auf weit mehr als nur basalen körperlichen Berührungsanwendungen beruhen. So gesehen haben auch Hebammen aufgrund ihrer intensiven Arbeit mit den Schwangeren, Gebärenden und Wöchnerinnen enorme Bedeutung für die prä-, periund postnatale Entwicklung.

Tatsächlich ist-angesichts der elementaren Bedeutung körperlich-seelischer Berührungen für das Immun- und Stresssystem - eine komplexere biopsychosoziale Wirkweise dieser Methoden anzunehmen, die auch beziehungsmedizinische Aspekte mitberücksichtigt [55]. Berührungsassoziierte Therapiemethoden verleihen der Behandler-Patienten-Beziehung Bedeutung und Tiefe und erzielen darüber wichtige therapeutische Effekte [31]. Jüngst wurden mit einem speziell für die Untersuchung komplexer Fragestellungen in der PNI entwickelten Forschungsansatz an einer 49-jährigen Brustkrebspatientin erstmals Hinweise für eine solche „Meaning Response“ [55] und die damit verbundene Entzündungshemmung bei Anwendung berührungsassoziierter Behandlungsmethoden nachgewiesen [76].

\section{FAZIT}

Über die gesamte Lebensspanne hinweg sind Berührungen als biopsychosoziales Geschehen von elementarer Bedeutung für Gesundheit und Krankheit.

Bereits vorgeburtliche Berührungserfahrungen moderieren maßgebend die biopsychosoziale Entwicklung eines Menschen. Gleichzeitig sind Berührungen untrennbar mit der für die Gesundheit so entscheidenden Bindungsentwicklung verbunden. Der Bindungsstil wiederum kann je nach Ausprägung - sicher, unsicher oder desorganisiert - einen Schutz- oder Risikofaktor darstellen [13]. Aktuelle Studien legen dar, dass positive Bindungsneuerfahrungen nicht nur auf Symptomebene eine „Normalisierung“ im Verhalten bewirken können, sondern auch das Nachreifen neuronaler Netzwerke in traumarelevanten kortikalen Strukturen. Diese Korrektur von fehlgeleiteten frühkindlichen Berührungs- und Bindungserfahrungen wurde bereits an schwer traumatisierten Kindern und Jugendlichen gezeigt [2][15][16][17]. Angesichts solcher Erkenntnisse lässt sich davon ausgehen, dass Berührungstraumen und der damit verbundene Schmerz überwunden werden können.

\section{Über diesen Artikel}

Dieser Beitrag ist eine Zweitveröffentlichung des Originalartikels: Ott M, Singer M, Brisch KH, Schubert C. Körperlich-seelische Berührungen im Fokus der Psychoneuroimmunologie. Der Schmerzpatient 2019; 2: 66-75

\section{Autorinnen / Autoren}

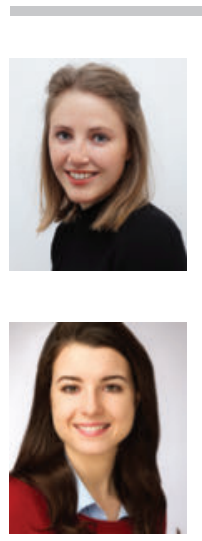

Michaela Ott studierte an der Leopold-Franzens-Universität Innsbruck Psychologie. Aktuell arbeitet sie an ihrer Promotion im Bereich der Psychoneuroimmunologie. Begleitend dazu ist sie im Raum München als Psychologin und Referentin für Gesundheitswissenschaften bei der BKK ProVita tätig.

Magdalena Singer ist Klinische- und Gesundheitspsychologin sowie Psychotherapeutin in Ausbildung. Im Rahmen ihrer Dissertation baut sie derzeit die Arbeitsgruppe Psychoneuroimmunologie an der Medizinischen Universität Wien auf. 


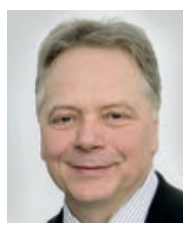

Professor Karl Heinz Brisch ist Leiter der Abteilung Pädiatrische Psychosomatik und Psychotherapie an der Kinderklinik und Poliklinik im Dr. von Haunerschen Kinderspital der Ludwig-Maximilians-Universität München, Lehranalytiker in Stuttgart sowie Facharzt für Psychosomatische Medizin und Psychotherapie und Neurologie. Er hat seit 2016 den weltweit ersten Lehrstuhl für Early Life Care inne und leitet das gleichnamige Forschungsinstitut an der Paracelsus Medizinischen Privatuniversität in Salzburg.

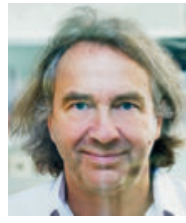

Professor Christian Schubert leitet das Labor für Psychoneuroimmunologie der Universitätsklinik für Medizinische Psychologie Innsbruck. Zudem ist er Leiter der Arbeitsgruppe ,Psychoneuroimmunologie' des Deutschen Kollegiums für Psychosomatische Medizin (DKPM) sowie Vorstandsmitglied der Thure von Uexküll-Akademie für Integrierte Medizin (AIM). Sein Forschungsschwerpunkt liegt auf der Entwicklung eines Forschungsansatzes zur Untersuchung von Komplexität in der

Psychoneuroimmunologie.

\section{Korrespondenzadresse}

\section{Christian Schubert}

Medizinische Universität Innsbruck

Department für Psychiatrie und Psychotherapie

Klinik für Medizinische Psychologie

Schöpfstraße 23a

6020 Innsbruck

Österreich

E-Mail: christian.schubert@i-med.ac.at

\section{Literatur}

[1] Akkaya H, Buke B. A frequently asked question: Is it normal not to feel my baby's movements yet? JCMA 2018; 81 (8): 742-746. doi:10.1016/j.jcma.2017.07.014

[2] Balkenhol C. Förderung der vorgeburtlichen Mutter-Kind-Bindung mit Hilfe der Bindungsanalyse nach Raffai und Hidas. In: Balkenhol C, Karrasch C, Hrsg. Mit deiner Liebe wächst meine Seele. Leben und Erleben im Mutterleib. Die Methode der vorgeburtlichen Bindungsförderung in Praxis und Theorie. Zetel: Einklang Verlag; 2017

[3] Balkenhol C. Was bedeutet Bindung und warum ist sie überlebenswichtig für das Neugeborene? Was kann die Förderung der vorgeburtlichen Bindung bewirken? In: Balkenhol C, Karrasch C, Hrsg. Mit deiner Liebe wächst meine Seele. Leben und Erleben im Mutterleib. Die Methode der vorgeburtliche Bindungsförderung in Praxis und Theorie. Zetel: Einklang Verlag; 2017

[4] Bauer J. Das Gedächtnis des Körpers. Wie Beziehungen und Lebensstile unsere Gene steuern. München: Piper, 2015

[5] Baumeister D, Akhtar R, Ciufolini $S$ et al. Childhood trauma and adulthood inflammation: a meta-analysis of peripheral Creactive protein, interleukin- 6 and tumour necrosis factoralpha. Molecular psychiatry 2016; 21 (5): 642-649. doi:10.1038/mp.2015.67

[6] Beijers R., Buitelaar JK, de Weerth C Carolina. Mechanisms underlying the effects of prenatal psychosocial stress on child outcomes: beyond the HPA axis. European child \& adolescent psychiatry 2014; 23 (10): 943-956. doi:10.1007/s00787-0140566-3

[7] Bernard K, Hostinar CE, Dozier M. Mary. Longitudinal associations between attachment quality in infancy, C-reactive protein in early childhood, and BMI in middle childhood: preliminary evidence from a CPS-referred sample. Attachment \& human development 2019; 21 (1): 5-22. doi:10.1080/14616734.2018.1541513

[8] Besedovsky HO, del Rey A. Physiology of psychoneuroimmunology: a personal view. Brain, behavior, and immunity 2007; 21 (1): 34-44. doi:10.1016/j.bbi.2006.09.008

[9] Bierhaus A, Wolf J, Andrassy M et al. A mechanism converting psychosocial stress into mononuclear cell activation. Proceedings of the National Academy of Sciences of the United States of America 2003; 100 (4): 1920-1925. doi:10.1073/ pnas. 0438019100

[10] Blalock JE. The syntax of immune-neuroendocrine communication. Immunology today 1994; 15 (11): 504-511

[11] Bowlby J. A secure base. Parent-child attachment and healthy human development. New York: Basic Books; 1988

[12] Bowlby J. Bindung. München, Basel: Ernst Reinhardt; 2006

[13] Brisch KH. SAFE ${ }^{\circledR}$ - Sichere Ausbildung für Eltern. Stuttgart: Klett-Cotta; 2010

[14] Brisch KH. Die Wiege der Sicherheit. Gehirn\&Geist 2011; (9): 48-55

[15] Brisch KH. Die transgenerationale Weitergabe von emotionaler Gewalt. In: Brisch KH,Hrsg. Bindung und emotionale Gewalt. Stuttgart: Klett-Cotta; 2017

[16] Brisch KH, Erhardt I, Kern C et al. How to treat children with severe attachment disorders after multiple early experiences of trauma? A model of treatment in an intensive care unit of psychotherapy. In: Barone L, Hrsg. 6th International Attachment Conference. Pianoro: Medimond; 2013

[17] Brisch KH, Oberschneider L, Hilmer $\mathrm{C}$ et al. The MOSES ${ }^{\circledR}$ Treatment Model: Efficacy and working mechanisms of treatment effects of children with attachment-traumatizations in infancy. Paper presented at the 16th WAIMH World Congress. Rom / Italien; 2018

[18] Brisch KH, Hilmer C, Oberschneider L et al. Bindungsstörungen. Monatsschrift Kinderheilkunde 2018; 166 (6): 533-544

[19] Bundesvertretung der Osteopathen in Deutschland. Was ist Osteopathie? Im Internet: https://www.osteopathie.de/osteo pathie-was_ist_osteopathie. Stand: 01.03.2019

[20] Buss C, Entringer S, Moog NK et al. Intergenerational Transmission of Maternal Childhood Maltreatment Exposure: Implications for Fetal Brain Development. Journal of the American Academy of Child and Adolescent Psychiatry; 201756 (5): 373-382. doi:10.1016/j.jaac.2017.03.001

[21] Cannon WB. Bodily changes in pain, hunger, fear and rage: An account of recent researches into the function of emotional excitement. New York: Appleton; 1915

[22] Cascio C], Moore D, McGlone F. Social touch and human development. Dev Cogn Neurosci. 2019; 35: 5-11. doi:10.1016/j. dcn.2018.04.009

[23] Coussons-Read ME, Okun ML, Nettles CD. Psychosocial stress increases inflammatory markers and alters cytokine production across pregnancy. Brain, behavior, and immunity 2007; 21 (3): 343-350. doi:10.1016/j.bbi.2006.08.006

[24] Danese A, Lewis S. Psychoneuroimmunology of Early-Life Stress: The Hidden Wounds of Childhood Trauma? Neuropsychopharmacology 2017; 42 (1): 99-114. doi:10.1038/ npp.2016.198 
[25] Dantzer R, O'Connor JC, Freund GG et al. From inflammation to sickness and depression: when the immune system subjugates the brain. Nature reviews neuroscience 2008; 9 (1): 46-56

[26] Duhn L. The importance of touch in the development of attachment. Advances in neonatal care: official journal of the National Association of Neonatal Nurses 2010; 10 (6): 294300. doi:10.1097/ANC.0b013e3181fd2263

[27] Dunkel Schetter C. Psychological science on pregnancy: stress processes, biopsychosocial models, and emerging research issues. Annual review of psychology 2011; 62: 531-558. doi:10.1146/annurev.psych.031809.130727

[28] Ehrlich KB, Katherine B. Attachment and psychoneuroimmunology. Current opinion in psychology 2018; 25: 96-100. doi:10.1016/j.copsyc.2018.03.012

[29] Elenkov II, Chrousos GP. Stress hormones, Th1 / Th2 patterns, pro/anti-inflammatory cytokines and susceptibility to disease. Trends in Endocrinology \& Metabolism 1999; 10 (9): 359-368

[30] Elenkov I], Chrousos GP. Stress system-organization, physiology and immunoregulation. Neuroimmunomodulation 2006; $13(5-6): 257-267$

[31] Elkiss ML, Jerome JA. Touch - more than a basic science. The Journal of the American Osteopathic Association 2012; 112 (8): 514-517

[32] Engel GL. The clinical application of the biopsychosocial model. American journal of Psychiatry 1980; 137: 535-544

[33] Entringer S, Kumsta R, Hellhammer DH et al. Prenatal exposure to maternal psychosocial stress and HPA axis regulation in young adults. Hormones and behavior 2009; 55 (2): 292298. doi:10.1016/j.yhbeh.2008.11.006

[34] Entringer S, Kumsta R, Nelson EL et al. Influence of prenata psychosocial stress on cytokine production in adult women. Developmental psychobiology 2008; 50 (6): 579-587. doi:10.1002/dev.20316

[35] Entringer S, Wust S, Kumsta R et al. Prenatal psychosocial stress exposure is associated with insulin resistance in young adults. American journal of obstetrics and gynecology 2008;199 (5): 498.e1-7. doi:10.1016/j.ajog.2008.03.006

[36] Felitti V], Anda RF, Nordenberg D et al. Relationship of childhood abuse and household dysfunction to many of the leading causes of death in adults. The Adverse Childhood Experiences (ACE) Study. American journal of preventive medicine 1998; 14 (4): 245-258

[37] Field T. Touch for socioemotional and physical well-being: A review. Developmental review 2010; 30 (4): 367-383

[38] Field T. Massage therapy research review. Complementary therapies in clinical practice 2016; 24: 19-31. doi:10.1016/j. ctcp.2016.04.005

[39] Glocker ML, Langleben DD, Ruparel K et al. Baby schema in infant faces induces cuteness perception and motivation for caretaking in adults. Ethology 2009; 115 (3): 257-263

[40] Grady MD, Yoder J, Brown A Adam. Childhood Maltreatment Experiences, Attachment, Sexual Offending: Testing a Theory. J Interpers Violence. 2018 Nov 25:886260518814262. doi: $10.1177 / 0886260518814262$. Epub ahead of print]

[41] Grunwald M. Der Tastsinn im Griff der Technikwissenschaften? Herausforderungen und Grenzen aktueller Haptikforschung. Hg. v. Lifis Online - Leibniz Institut 2009. Im Internet: https://leibniz-institut.de/archiv/grunwald_mar tin_09_01_09.pdf. Stand: 01.03.2019

[42] Gunnar MR, Hostinar CE, Camelia E. The social buffering of the hypothalamic-pituitary-adrenocortical axis in humans: Developmental and experiential determinants. Social neuroscience 2015: 10 (5): 479-488. doi:10.1080/ 17470919.2015.1070747

[43] Gunnar MR, Mangelsdorf S, Larson M et al. Attachment, temperament, and adrenocortical activity in infancy: A study of psychoendocrine regulation. Developmental Psychology 1989; 25 (3): 355

[44] Hellbrügge T. Zur Problematik der Säuglings- und Kleinkinderfürsorge in Anstalten, Hospitalismus und Deprivation. In: $\mathrm{H}$. Opitz $\mathrm{H}$ und F. Schmid F (Hg.). Handbuch der Kinderheilkunde. Berlin, Heidelberg, New York: Springer; 1966

[45] Jones GT, Power C, Macfarlane GJ. Adverse events in childhood and chronic widespread pain in adult life: Results from the 1958 British Birth Cohort Study. Pain 2009; 143 (1-2): 92-96. doi:10.1016/j.pain.2009.02.003

[46] Kiecolt-Glaser JK, Gouin JP, Hantsoo L. Close relationships, inflammation, and health. Neuroscience and biobehavioral reviews 2010; 35 (1): 33-38. doi:10.1016/j. neubiorev.2009.09.003

[47] Landmann RM, Muller FB, Perini C et al. Changes of immunoregulatory cells induced by psychological and physical stress: relationship to plasma catecholamines. Clinical and experimental immunology 1984; 58 (1): 127-135

[48] Lee Masson H, Beeck $\mathrm{H}$ op de. Socio-affective touch expression database. PloS one 2018; 13 (1), e0190921. doi:10.1371/journal.pone.0190921

[49] Licciardone JC, Kearns CM, Hodge LM et al. Associations of cytokine concentrations with key osteopathic lesions and clinical outcomes in patients with nonspecific chronic low back pain: results from the Osteopathic Trial. The Journal of the American Osteopathic Association 2012; 112 (9): 596-605

[50] Liotti G. Trauma, dissociation, and disorganized attachment: Three strands of a single braid. Psychotherapy: Theory, research, practice, training 2004; 41 (4): 472

[51] Liu J, Chen X, Wang X. Ethical issues in neonatal intensive care units. The Journal of Maternal-Fetal \& Neonatal Medicine 2015; 29 (14): 2322-2326. doi:10.3109/ 14767058.2015.1085016

[52] Measelle JR, David J, Ablow JC. Increased levels of inflammation among infants with disorganized histories of attachment. Behavioural brain research 2017; 325 (Pt B): 260-267. doi:10.1016/j.bbr.2016.12.001

[53] Merlot E, Couret D, Otten W. Prenatal stress, fetal imprinting and immunity. Brain, behavior, and immunity 2008; 22 (1): 42-51. doi:10.1016/j.bbi.2007.05.007

[54] Mierau S. Geborgen Wachsen. Bindung durch Berührung Mein Vortrag auf dem Attachment Parenting Kongress 2014. Im Internet: https://geborgen-wachsen.de/2014/10/17/bin dung-durch-beruehrung-mein-vortrag-auf-demattachment-p arenting-kongress/. Stand: 03.03.2019

[55] Moerman DE, Jonas WB. Deconstructing the placebo effect and finding the meaning response. Annals of internal medicine 2002; 136 (6): 471-476

[56] Moog NK, Entringer S, Rasmussen JM et al. Intergenerational Effect of Maternal Exposure to Childhood Maltreatment on Newborn Brain Anatomy. Biological psychiatry 2018; 83 (2): 120-127. doi:10.1016/j.biopsych.2017.07.009

[57] Morrison I. Keep calm and cuddle on: social touch as a stress buffer. Adaptive Human Behavior and Physiology 2016; 2: 344-362

[58] Muehsam D, Lutgendorf S, Mills P] et al. The embodied mind: A review on functional genomic and neurological correlates of mind-body therapies. Neuroscience and biobehavioral reviews 2017; 73: 165-181. doi:10.1016/j.neubiorev.2016.12.027 
[59] Nacak Y, Morawa E, Tuffner D. Insecure attachment style and cumulative traumatic life events in patients with somatoform pain disorder: A cross-sectional study. Journal of Psychosomatic Research 2017; 103: 77-82. doi:10.1016/j. jpsychores.2017.10.003

[60] Nixdorff S. Salutogenese und Pränatale Psychologie. Gesundheitsförderung und Prävention in der vorgeburtlichen Lebensspanne. Aktualisierter Nachdruck. Heidelberg: Mattes; 2010

[61] Obegi JH. The development of the client-therapist bond through the lens of attachment theory. Psychotherapy: Theory, research, practice, training 2008; 45 (4): 431

[62] O'Donnell K, O'Connor TG, Glover V. Prenatal stress and neurodevelopment of the child: focus on the HPA axis and role of the placenta. Developmental neuroscience 2009; 31 (4): 285-292. doi:10.1159/000216539

[63] Ott M, Singer M, Schubert C. Psychoneuroimmunologie im pränatalen Raum. Wie psychosoziale Belastungen in der Schwangerschaft das Stresssystem des Kindes langfristig beeinträchtigen. Kinderärzte Schweiz 2018; (03): 27-31

[64] Pfaundler M von. Die Physiologie des Neugeborenen. In: Döderlein A, Hrsg. Handbuch der Geburtshilfe. Wiesbaden: Bergmann; 1915

[65] Pietromonaco PR, Powers SI. Attachment and Health-Related Physiological Stress Processes. Current opinion in psychology 2015; 1: 34-39. doi:10.1016/j.copsyc.2014.12.001

[66] Puig J, Englund MM, Simpson JA et al. Predicting adult physical illness from infant attachment: a prospective longitudinal study. Health psychology: official journal of the Division of Health Psychology, American Psychological Association 2013; 32 (4): 409-417. doi:10.1037/a0028889

[67] Ross KM, Miller G, Qadir S et al. Close relationship qualities and maternal peripheral inflammation during pregnancy. Psychoneuroendocrinology 2017; 77: 252-260. doi:10.1016/j. psyneuen.2017.01.003

[68] Schieche M, Spangler G. Individual differences in biobehavioral organization during problem-solving in toddlers: the influence of maternal behavior, infant-mother attachment, and behavioral inhibition on the attachment-exploration balance. Developmental psychobiology 2005; 46 (4): 293-306. doi:10.1002/dev.20065

[69] Schleußner E. Fetale Programmierung. In: Schneider H, Husslein P, Schneider KTM, Hrsg. Die Geburtshilfe. Berlin, Heidelberg: Springer; 2016

[70] Schmid W. Mit sich selbst befreundet sein. Von der Lebenskunstim Umgang mit sich selbst. Frankfurt am Main: Suhrkamp; 2004

[71] Schmid W. So viele Arten, einander zu berühren. In: Neues Deutschland - Sozialistische Tageszeitung, 2005. Im Internet: https://www.neues-deutschland.de/artikel/72841.so-vielear ten-einander-zu-beruehren.html . Stand: 03. 03.2019

[72] Schrey S, Stepan H. Pränatale epigenetische Prägung: Stand des Wissens. Dtsch Arztebl International 2016; 113 (45): A-2040

[73] Schröder H. Das Nocebophänomen - Wie Kommunikation krank machen kann. In: Erfahrungsheilkunde 2016; 48 (2): 84-89

[74] Schubert C. Ein Blick durch die biopsychosoziale Brille. Warum Immunologie und Psychotraumatologie immer näher zusammenrücken. Jung-Journal 2016; 36: 29-35

[75] Schubert C, Exenberger S. Einfluss von frühen psychischen Belastungen auf die Entwicklung von Entzündungserkrankungen im
Erwachsenenalter. In: Schubert C, Hrsg. Psychoneuroimmunologie und Psychotherapie. Stuttgart: Schattauer; 2015

[76] Schubert C, Ott M, Hannemann J et al. Psychoimmunological effects of complementary and alternative medicine techniques: An integrative single-case study on a breast cancer survivor. (Zur Publikation eingereicht)

[77] Schubert C. Einführung. In: Schubert C, Hrsg. Psychoneuroimmunologie und Psychotherapie. Stuttgart: Schattauer; 2015

[78] Schubert C. Psychoneuroimmunologie körperlicher Erkrankungen. In: Schubert C, Hrsg. Psychoneuroimmunologie und Psychotherapie. Stuttgart: Schattauer; 2015

[79] Schubert C. Bewusstwerdung als Heilung - die Wirkung künstlerischen Tuns auf das Immunsystem. In: Spreti F von, Martius P, Steger F, Hrsg. KunstTherapie. Stuttgart: Schattauer; 2017

[80] Seckl JR, Cleasby M, Nyirenda M]. Glucocorticoids, 11ß-hydroxysteroid dehydrogenase and fetal programming. Kidney International 2000, 57 (4): 1412-1417. doi:10.1046/j.15231755.2000.00984.x

[81] Seckl JR, Holmes MC. Mechanisms of disease: glucocorticoids, their placental metabolism and fetal ,programming of adult pathophysiology. Nature Reviews Endocrinology 2007; 3 (6): 479

[82] Selim M, Riepl G. Was kann die Osteopathie und wie heilt sie? Die Kunst des Osteopathen besteht darin, jeden Patienten und sein Problem als einzigartig zu betrachten. ProMed komplementär 2009; 16 (2): 20-23

[83] Sherer ML, Posillico CK, Schwarz JM. The Psychoneuroimmunology of Pregnancy. Front Neuroendocrinol. 2018; 51: 2535. doi:10.1016/j.yfrne.2017.10.006

[84] Spitz R. Hospitalism. An Inquiry into the Genesis of Psychiatric Conditions in Early Childhood. In: The Psychoanalytic Study of the Child 1 1945; (01): 53-74. doi:10.1080/ 00797308.1945 .11823126

[85] Tracey KJ. The inflammatory reflex. Nature 2002; 420 (6917): 853-859

[86] Tsigos C, Chrousos GP. Hypothalamic-pituitary-adrenal axis, neuroendocrine factors and stress. Journal of Psychosomatic Research 2002; 53 (4): 865-871

[87] Wadhwa PD. Psychoneuroendocrine processes in humapregnancy influence fetal development and health. Psychoneuroendocrinology 2005; 30 (8): 724-743. doi:10.1016/j. psyneuen.2005.02.004

[88] World Osteopathic Health Organization. What is Osteopathy? Im Internet: http://www.woho.org/what-is-osteopathy-2/ Stand: 03.03.2019

[89] Zouikr I, Karshikoff B. Lifetime Modulation of the Pain System via Neuroimmune and Neuroendocrine Interactions. Frontiers in immunology 2017; 8: 276. doi:10.3389/fimmu.2017.00276

\section{Bibliografie}

DOI https://doi.org/10.1055/a-1084-5241

Die Hebamme 2020; 33: 48-57

(c) Georg Thieme Verlag KG Stuttgart · New York

ISSN 0932-8122 